\title{
BMJ Open Development of a national neonatal intensive care unit-specific antimicrobial stewardship programme in Canada: protocol for a cohort study
}

\author{
Joseph Y. Ting (D) , ${ }^{1}$ Ashley Roberts, ${ }^{1}$ Peter Tilley, ${ }^{2}$ Joan L Robinson (D) , ${ }^{3}$ \\ Michael S Dunn, ${ }^{4}$ Vanessa Paquette, ${ }^{5}$ Kyong-Soon Lee, ${ }^{4}$ Vibhuti Shah, ${ }^{4}$ \\ Eugene Yoon, ${ }^{6}$ Lindsay L Richter, ${ }^{1}$ Abhay Lodha, ${ }^{7}$ Sandesh Shivananda, ${ }^{1}$ \\ Nisha Thampi, ${ }^{8}$ Julie Autmizguine, ${ }^{9}$ Prakesh S Shah, ${ }^{4,6}$ for the Canadian Neonatal \\ Network Investigators
}

To cite: Ting JY, Roberts A, Tilley $\mathrm{P}$, et al. Development of a national neonatal intensive care unit-specific antimicrobial stewardship programme in Canada: protocol for a cohort study. BMJ Open 2020;10:e043403. doi:10.1136/ bmjopen-2020-043403

- Prepublication history for this paper is available online. To view these files, please visit the journal online (http://dx.doi. org/10.1136/bmjopen-2020043403).

Received 03 August 2020 Revised 23 November 2020 Accepted 27 November 2020

Check for updates

(c) Author(s) (or their employer(s)) 2020. Re-use permitted under CC BY-NC. No commercial re-use. See rights and permissions. Published by BMJ.

For numbered affiliations see end of article.

Correspondence to Dr Joseph Y. Ting; jting2@cw.bc.ca

\section{ABSTRACT}

Introduction Early empiric treatment with broadspectrum antimicrobials is common in neonatal intensive care units (NICU) due to the non-specific clinical presentation of infection. However, excessive and inappropriate antimicrobial use can lead to the emergence of drug-resistant organisms and adverse neonatal outcomes. This study aims to develop and implement a nationwide NICU-specific antimicrobial stewardship programme (ASP) to promote judicious antimicrobial use and control the emergence of multidrug-resistant organisms (MDROs) in Canada.

Methods and analysis Our study population will include all very low-birth-weight neonates admitted to participating tertiary NICU in Canada. Based on the existing limited literature, we will develop consensus on NICU antimicrobial stewardship interventions to enhance best practices. Using an expanded Canadian Neonatal Network (CNN) platform, we will collect data on antimicrobial use and the susceptibility of organisms identified in clinical samples from blood and cerebrospinal fluid over a period of 2 years. These data will be used to provide all NICU stakeholders with benchmarked centre-adjusted antimicrobial use and MDRO prevalence reports. An ASP plan will be developed at both individual unit and national levels in the subsequent years. Knowledge translation strategies will be implemented through the wellestablished Evidence-based Practice for Improving Quality methodology.

Ethics and dissemination Ethics for the study has been granted by the University of British Columbia Children's \& Women's Research Ethics Board (H19-02490) and supported by CNN Executive Committee. The study results will be disseminated through national organisations and open access peer-reviewed publications.

Trial registration number NCT04388293.

\section{INTRODUCTION}

The neonatal intensive care unit (NICU) is a unique clinical environment that houses neonates who are highly susceptible to
Strengths and limitations of this study

- Our study will identify key areas to target appropriate antimicrobial stewardship strategies and best evidence-based practices at both national and individual neonatal intensive care unit (NICU) levels.

- We are enrolling infants born at $<1500 \mathrm{~g}$, who are at highest risk of developing sepsis, across the majority of tertiary Canadian NICUs.

- Our study addresses the priorities of antimicrobial resistance research and rapid implementation of a NICU-specific antimicrobial stewardship programme nationally by using a well-defined knowledge translation (KT) plan and comprehensive engagement of key stakeholders.

- Practice changes during the KT plan implementation may require a high level of change in antimicrobial prescription or multidrug-resistant organisms screening, in addition to the resources and barriers required for audit and feedback services. Such changes may result in behaviour shifts within the working environment that may be met with resistance.

overwhelming infections, which can potentially result in significant neonatal morbidity and mortality. Many admitted neonates are preterm and/or have very low-birth-weight (VLBW; birth weight $<1500 \mathrm{~g}$ ), placing them at even greater risk for infection. ${ }^{12}$ Because the features of infection in neonates are nonspecific, early empiric treatment with antimicrobials is common practice in the NICU. ${ }^{3}$ However, there are no widely accepted guidelines regarding the choice and duration of antimicrobials for common neonatal conditions. ${ }^{4}$ Antimicrobials are often prescribed and continued in clinical situations in which a clear indication or benefit has not been demonstrated. ${ }^{5}$ Excessive and inappropriate 
antimicrobial use can lead to gut dysbiosis, emergence of drug-resistant organisms and adverse neonatal outcomes. ${ }^{6-12}$

Antimicrobial stewardship programmes (ASPs) are designed to optimise clinical outcomes while mitigating unintended consequences of antimicrobial misuse and control the emergence of multidrug-resistant organisms (MDROs). ${ }^{13}$ ASPs include active monitoring of antimicrobial resistance, promotion of appropriate antimicrobial use, and collaboration with infection prevention and control programme departments to minimise the spread of resistance. ${ }^{14}$

We recently identified significant site variations in the antimicrobial utilisation rate (AUR) among neonates born at $<33$ weeks of gestational age in NICUs in Canada. ${ }^{15} \mathrm{~A}$ study in a tertiary NICU in Vancouver, British Columbia, found that $11.4 \%-26.3 \%$ of broad-spectrum antimicrobial use was inappropriate. ${ }^{16}$ Although some NICUs have ASPs, there are no guidelines in Canada or elsewhere for NICU-specific antimicrobial stewardship best practices and strategies. ${ }^{51417}$ We are not aware of any validated antimicrobial usage guidelines that address the unique challenges faced in the management of NICU patients, such as culture-negative clinical sepsis and empiric treatment of early-onset sepsis. ${ }^{18}{ }^{19}$ Moreover, there are no established sets of evidence-based NICU-specific metrics to measure the success or failure of ASP initiatives. ${ }^{18}$ Recent systemic review aiming to understand the effectiveness of ASPs revealed successful reduction of antibiotic consumption and/or inappropriate antibiotic use in four studies, but none has been identified to evaluate the impact of ASPs on antimicrobial resistance or the incidence of nosocomial infection in neonates. ${ }^{20}$

Our overarching goal is to develop and implement a NICU-specific ASP at the individual unit and national levels within Canada to promote optimal antimicrobial utilisation and decrease MDRO infections in this specialised population.

\section{METHODS AND ANALYSIS}

Starting from 2020, we will conduct a 5-year cohort study to address three specific aims (figure 1):

Aim 1: To gather baseline information, build capacity for data (data collection, analysis and reporting), and engage Canadian NICU key stakeholders (years 1-5).

Aim 2: To develop evidence-based NICU-specific ASP principles and practices at the national and individual unit levels (years 1-4).

Aim 3: To evaluate the effect of ASP implementation (years 2-5)

\section{Aim 1: to gather baseline information, build capacity for data and engage key stakeholders (years 1-5)}

We will collect data on antimicrobial use (drug and duration) and the susceptibility of organisms identified in clinical samples obtained from NICU patients using the Canadian Neonatal Network (CNN) platform, which collects demographic and neonatal outcome data for $>90 \%$ of VLBW neonates in Canada. NICU stakeholders will be provided with benchmarked centre-adjusted antimicrobial use and MDRO prevalence reports on a yearly basis to create awareness of the problem and its magnitude. Examples of MDRO include methicillin-resistant Staphylococcus aureus, vancomycin-resistant Enterococcus, Gram-negative bacilli resistant to third-generation and fourth-generation cephalosporins and/or carbapenem groups. Simultaneously, we will develop consensus-based practices of NICU antimicrobial stewardship interventions at the start of the programme (year 1). This will be

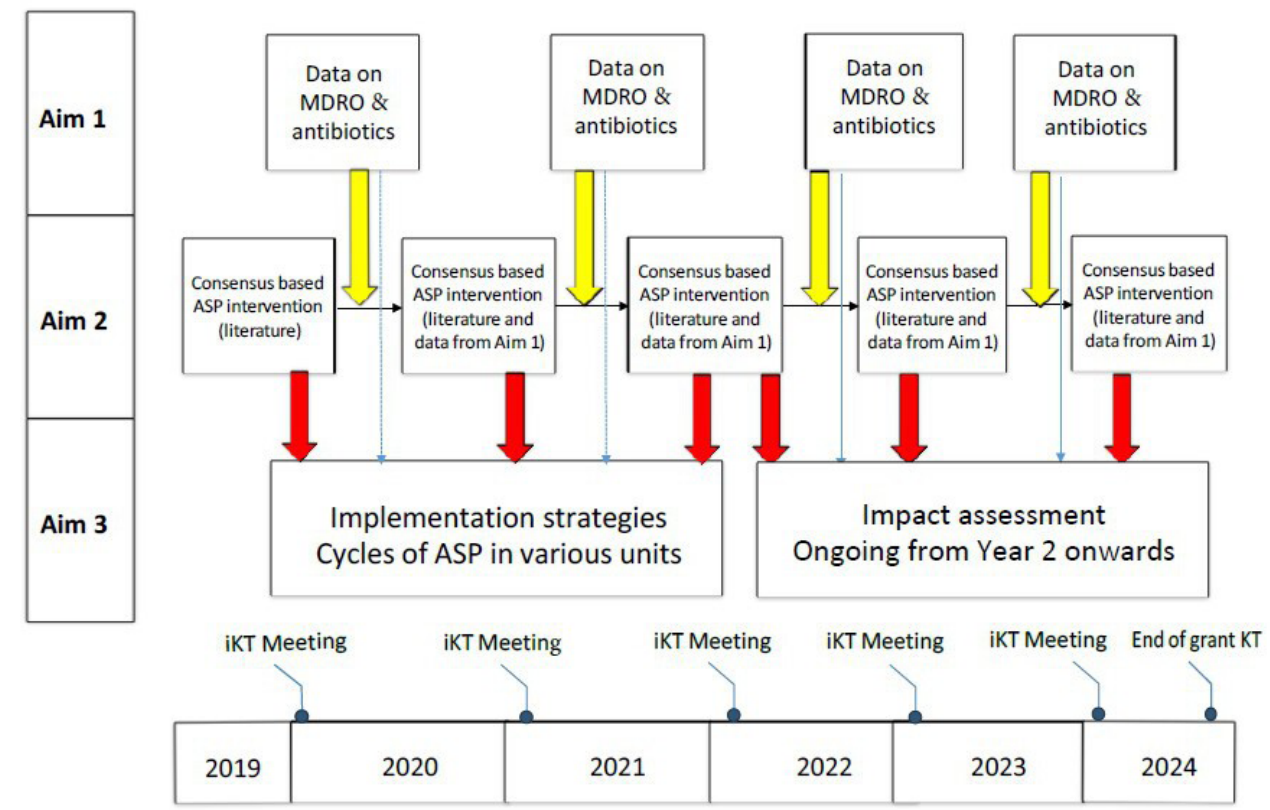

Figure 1 Approximate timeline of national neonatal intensive care unit-specific ASP plan. ASP, antimicrobial stewardship programme; iKT, integrated knowledge translation; MDRO, multidrug-resistant organism. 
based on the review of existing literature by a group of experts from neonatology, paediatric infectious disease, microbiology, infection control, clinical pharmacy and epidemiology from various institutes across the country. Baseline information will help inform development of the NICU-specific ASPs in Aim 2.

\section{Hypotheses}

We hypothesise that we will find:

- Significant variation in broad-spectrum antimicrobial usage across NICUs, but no correlation with the incidence of culture-proven bacteraemia and/or meningitis. Higher use of antimicrobials will be associated with limited existing ASP resources and increased neonatal morbidities related to inflammatory cascades $^{11} 21$ (bronchopulmonary dysplasia, retinopathy of prematurity and periventricular leucomalacia).

- Significant variations in the prevalence of MDRO across NICUs.

- Differences in empiric antimicrobial regimens that may be associated with MDRO prevalence, and these variations in antimicrobial use could explain some of the variations in neonatal morbidities.

\section{Methods}

Our study population will include all VLBW neonates admitted to participating tertiary NICUs in Canada. All VLBW neonates will be included in the primary MDRO prevalence analysis; but for the analysis of the effect of antimicrobial use and MDRO on neonatal outcomes, we will exclude neonates with major congenital anomalies in order to avoid the effect of underlying conditions on outcomes. ${ }^{22}$ In $\mathrm{CNN}$, data are recorded from $~ 3000$ VLBW neonates each year, of whom 500-600 develop culture-proven sepsis (bacteraemia or meningitis). ${ }^{23-26}$ Thus, we expect $\sim 6000$ VLBW neonates during the 2-year surveillance period of the study. This will provide us with 2-year surveillance data for the development of ASP planning at both individual unit and national levels in the subsequent years. Previous studies that have investigated broad-spectrum antimicrobial use in NICUs have had limited sample sizes, and reports of the infection and colonisation rates with MDRO have varied significantly among these studies. ${ }^{27-30}$

The primary outcomes are (1) the days of therapy (DOT)/1000 patient-days of antimicrobial use and (2) the prevalence of MDRO in infections in VLBW neonates during their NICU admission. The secondary outcomes include the association between MDRO, antimicrobial use and MDRO screening policies with neonatal outcomes. Simple and adjusted comparisons using propensity score will be performed.

\section{Analyses}

Comparisons of patient characteristics, antimicrobial utilisation and prevalence of MDRO across different sites will be performed using descriptive statistics and kernel density estimation. Regression analyses will be conducted to determine the association between antimicrobial utilisation and neonatal outcomes after controlling for confounding characteristics (such as gestational age, sex, small-for-gestational age and Score for Neonatal Acute Physiology). We will develop generalised linear models with the appropriate link functions and adjust for potential confounders. Spearman or linear correlation will be applied to assess the relationship between broadspectrum antimicrobial use and the prevalence of MDRO in individual NICUs.

\section{Aim 2: to develop evidence-based NICU-specific ASP metrics} to evaluate the effectiveness of ASP strategies at the individual unit and national levels (years 1-4)

To address this aim, two stages are planned: (1) development of the first set of guidelines based on existing literature and consensus at the outset of the project and (2) revision of these guidelines informed by our national data.

After obtaining baseline data in Aim 1 (overall AUR and DOT of specific antimicrobials, and MDRO epidemiology in each unit), benchmarking of these data across Canada will be performed. Stakeholders and knowledge users will meet to revise strategies and potential best practices to target specific challenges at both the unit and national levels. Examples include implementation of specific guideline and strategies to reduce vancomycin and third-generation cephalosporin use, and use of 36-hour automatic stop for empirical antimicrobial coverage (table 1). ${ }^{43132}$

In general, we aim to reduce prolonged empirical antibiotic use for $>3$ days in the first week of life among infants at very low risk of early-onset sepsis (eg, elective caesarean section for maternal or fetal indication) by $80 \%$ across Canada and reduce broad-spectrum antimicrobial use in this population by $25 \%$ across Canada, after 2 years. Other specific targets will be determined once surveillance data become available.

\section{Integrated quality improvement and knowledge translation/ implementation}

We will apply the Evidence-based Practice for Improving Quality (EPIQ) method, a proven knowledge translation (KT) strategy derived from the 'Promoting Action on Research Implementation in Health Services' framework. This methodology examines the interaction between evidence, context and facilitation for implementation of research into practice. ${ }^{33} 34$ The EPIQ principles include (1) identifying site-specific associations between practices and outcomes through baseline data analysis; (2) involving NICU teams in systematic evidence reviews; (3) identifying barriers to change; (4) developing evidence-based, site-specific priorities and practice-change strategies; (5) monitoring compliance and (6) providing tailored support for continuous quality improvement (QI) using the Plan-Do-Study-Act model. 
Table 1 Specific antimicrobial stewardship programme strategies to reduce inappropriate antimicrobial use

\begin{tabular}{|c|c|c|}
\hline Problem & Possible strategies & Reference \\
\hline $\begin{array}{l}\text { High prevalence of vancomycin-resistant } \\
\text { enterococci or vancomycin usage }\end{array}$ & $\begin{array}{l}\text { Vancomycin reduction guideline to cut } \\
\text { down the overuse }\end{array}$ & Chiu et al. ${ }^{31}$ \\
\hline High broad-spectrum antibiotic usage & $\begin{array}{l}\text { Reduce use of broad-spectrum } \\
\text { perioperative antibiotic prophylaxis for } \\
\text { clean surgical procedures; } \\
\text { avoid prolonged duration of postoperative } \\
\text { prophylaxis days of therapy of perioperative } \\
\text { prophylaxis > } 48 \text { hours; } \\
\text { avoid prolonged duration of culture- } \\
\text { negative sepsis }\end{array}$ & Cantey et al. ${ }^{4}$ \\
\hline
\end{tabular}

\section{KT plan/dissemination of surveillance results (Aims 1 and 2)}

Key messages from study results will be disseminated to relevant stakeholders (including parents, professional organisations and policymakers) and future research directions will be generated. We will disseminate our findings through (1) ASP practice guidelines, yearly reports, and annual meetings of CNN and Canadian Paediatric Society and (2) open access peer-reviewed publications, media releases and interprofessional co-presentations at national and international meetings. Online ASP teaching to knowledge users will be developed. ${ }^{35}$ We will provide executive summaries to provincial and national organisations (eg, Provincial Council for Maternal and Child Health and Canadian Paediatric Society) for consideration of production of national best practice guidelines.

\section{Aim 3: to evaluate the effectiveness of the ASP (years 2-5)}

We will analyse the effects of adoption of an ASP in individual units on antimicrobial use and MDRO incidence by creating control charts. Implementation fidelity and transportable lessons of implementing antimicrobial stewardship interventions across multiple NICUs in changing real-world setting will be assessed using the learning evaluation method.

\section{Hypotheses}

- The overall usage across the country and in the units with initially high AUR will significantly decrease.

- The prevalence of 'clinical sepsis' (defined as use of at least 5 days of antimicrobials with no detection of pathogens) ${ }^{2636}$ will decrease.

- More units will adopt the screening policies for MDRO, especially among those who have higher MDRO prevalence than the national average.

- The prevalence of MDRO will remain the same or decrease in some units; this can be attributed to reduction of broad-spectrum antimicrobial consumption and enhanced screening policies.

- The prevalence of neonatal morbidities, such as necrotising enterocolitis may decrease in units with reduction of broad-spectrum antimicrobial usage.

\section{Methods}

Stewardship interventions should be sustainable and aligned with the available local resources. We will monitor the effectiveness (eg, reduction of antimicrobial use in neonates without culture-proven sepsis) and safety (eg, recurrence of infections shortly after stopping antimicrobials) of the new ASP potential best practices through regular evaluation of outcomes among knowledge users (such as quarterly teleconferences, during annual CNN meetings and annual Canadian Paediatric Society meetings). Along with the CNN report, we will publish an annual report of potential safety and quality metrics of ASP (eg, usage of broad-spectrum antimicrobials, rates of infections with MDRO, duration of treatment for culturenegative clinical sepsis and recurrence of bacteraemia). We will hold annual face-to-face meetings similar to that of the planning meeting for Aim 2 to address concerns from knowledge users in our research plan and review ASP strategies on a yearly basis, timed around CNN meetings.

\section{Analyses plan}

We will perform descriptive analyses to describe the prevalence of antimicrobial prescription and MDRO. Student's t-test, Wilcoxon rank-sum and $\chi^{2}$ tests will be used, as appropriate, to compare demographic and outcome characteristics between the baseline and intervention periods. Multivariate analysis can be performed through (1) logistic regression, based on findings of the univariate analysis and adjusted for potential confounding variables, to determine the factors associated with increased antimicrobial consumption and MDRO prevalence; (2) propensity score-based analyses to estimate the effect after discarding all unmatched units (ie, difference in baseline characteristics); (3) instrumental variable analyses to control for confounding and measurement errors in the observations before and after interventions, so that causal inferences can be made; and (4) interrupted time series analyses to establish whether there is a change in antimicrobial consumption during the study period. 


\section{Patient and public involvement}

This is a prospectively designed retrospective cohort study, and all infants are following the standard of clinical care per their unit policy. The necessity for rapid implementation of NICU-specific ASP nationally has emerged as an urgent priority. Our study addresses both the Public Health Agency of Canada's call to take action through new investment in antimicrobial resistance research and the research priority of the Canadian Institutes of Health Research Institute of Infection and Immunity. Parents were not directly involved in the proposal development; however, as highlighted in our protocol (Aim 2), family advisers would be invited to join our KT activity, to provide their thoughts on the best antimicrobial practice at both local and national levels after obtaining 2-year baseline data. The results will be disseminated to the public (parents) through media releases and open access publications.

\section{DISCUSSION}

This study will help identify key areas of inappropriate antimicrobial use and the prevalence of MDRO in Canadian NICUs. We will then develop appropriate ASP strategies and potential best practices at both individual NICU units and national levels to reduce unnecessary broadspectrum antimicrobial use, decrease the prevalence of MDRO and improve neonatal outcomes.

We anticipate challenges during the study. First, there may be variations in the blood culture collection practices in different hospitals (eg, one versus two vials collected or use of cord blood for initial blood cultures) and in the breakpoints used for antimicrobial susceptibility testing across different microbiology laboratories. These could result in some variations in bacteraemia and MDRO rates. Although it is virtually impossible to standardise all practices at the national level, we will conduct surveillance on these aspects in an attempt to understand the reasons for practices and address the potential impact of these variations on our results.

Second, clinicians may not be receptive to the results of comparative analyses. Many factors underlie the variations in antimicrobial prescription practices, prevalence of MDRO and neonatal outcomes in each NICU; thus, potential users may disregard findings. The recommended practice changes may require a major change in antimicrobial prescription or MDRO screening. There are major resources required to perform the interventions, such as audit and feedback. To address these challenges, we will use the national collaboration with EPIQ in CNN, which has been applied for $>10$ years. This national collaboration has created a clinical culture of embracing change and QI, which has led to a reduction in nosocomial infections and bronchopulmonary dysplasia rates, ${ }^{37}$ and we are hopeful for similar success in this project.

The promotion of appropriate antimicrobial usage has gained traction as a critical tool against the emergence of MDRO and is an important metric for patient safety and quality. This study will address existing knowledge gaps and facilitate the development of a standardised set of evidence-based NICU-specific ASP strategies to improve neonatal outcomes.

\section{ETHICS AND DISSEMINATION}

Ethics for the study has been granted by the University of British Columbia Children's \& Women's Research Ethics Board (H19-02490) and supported by CNN Executive Committee. The study results will be disseminated through (1) ASP practice guidelines, yearly reports and annual meetings of CNN and Canadian Paediatric Society and (2) open access peer-reviewed publications, media releases and inter-professional co-presentations at national and international meetings.

\section{Author affiliations}

${ }^{1}$ Department of Pediatrics, University of British Columbia, Vancouver, British Columbia, Canada

${ }^{2}$ Pathology and Laboratory Medicine, University of British Columbia, Vancouver, British Columbia, Canada

${ }^{3}$ Department of Pediatrics, University of Alberta, Edmonton, Alberta, Canada

${ }^{4}$ Department of Pediatrics, University of Toronto, Toronto, Ontario, Canada

${ }^{5}$ School of Pharmaceutical Science, University of British Columbia, Vancouver, British Columbia, Canada

${ }^{6}$ Maternal-Infant Care Research Centre, Mount Sinai Hospital, Toronto, Ontario, Canada

${ }^{7}$ Department of Pediatrics, University of Calgary, Calgary, Alberta, Canada ${ }^{8}$ Department of Pediatrics, University of Ottawa, Ottawa, Ontario, Canada ${ }^{9}$ Department of Pediatrics, University of Montreal, Montreal, Québec, Canada

Acknowledgements Co-applicants in this Canadian Institutes of Health Research project grant application (British Columbia: AR, PT, VP, Sarka Lisonkova and SS; Alberta: JR and AL; Ontario: MSD, K-SL, VS and NT; Newfoundland and Labrador: Akhil Deshpandey; Quebec: JA and Canadian Neonatal Network site investigators and data abstractors)

Contributors Conception and design of the work: JT and PSS. Development of the original research protocol and grant: JT (nominated principal applicant), AR, PT, JLR, MSD, VP, K-SL, VS, EY, LLR, AL, SS, NT, JA and PSS (principal applicant). Writing up the first drafting of this manuscript: JT. Critical revision of the manuscript for important intellectual content: JT, AR, PT, JLR, MSD, VP, K-SL, VS, EY, LLR, AL, SS, NT, JA and PSS. Approval of the submitted manuscript: JLR, MSD, VP, K-SL, VS, EY, LLR, AL, SS, NT, JA and PSS.

Funding This work was supported by the Canadian Institutes of Health Research (201903PJT-420294-CA2-CAAA-245530), which has no role in the design of the study and the writing of manuscript.

Competing interests JT is supported by the Investigator Grant Award Program through the British Columbia Children's Hospital Research Institute. Dr Prakesh S Shah reported holding an Applied Research Chair in Reproductive and Child Health Services and Policy Research awarded by the Canadian Institutes of Health Research (grant APR-126340).

Patient and public involvement Patients and/or the public were not involved in the design, or conduct, or reporting, or dissemination plans of this research.

Patient consent for publication Not required.

Provenance and peer review Not commissioned; externally peer-reviewed.

Open access This is an open access article distributed in accordance with the Creative Commons Attribution Non Commercial (CC BY-NC 4.0) license, which permits others to distribute, remix, adapt, build upon this work noncommercially, and license their derivative works on different terms, provided the original work is properly cited, appropriate credit is given, any changes made indicated, and the use is non-commercial. See: http://creativecommons.org/ licenses/by-nc/4.0/. 


\section{ORCID iDs}

Joseph Y. Ting http://orcid.org/0000-0002-5246-8823

Joan L Robinson http://orcid.org/0000-0001-9831-5681

\section{REFERENCES}

1 Hornik $\mathrm{CP}$, Fort $\mathrm{P}$, Clark $\mathrm{RH}$, et al. Early and late onset sepsis in verylow-birth-weight infants from a large group of neonatal intensive care units. Early Hum Dev 2012;88 Suppl 2:S69-74.

2 Camacho-Gonzalez A, Spearman PW, Stoll BJ. Neonatal infectious diseases: evaluation of neonatal sepsis. Pediatr Clin North Am 2013:60:367-89.

3 Mukhopadhyay S, Puopolo KM. Antibiotic use and mortality among premature infants without confirmed Infection-Perpetrator or innocent bystander? JAMA Pediatr 2016;170:1144-6.

4 Cantey JB, Patel SJ. Antimicrobial stewardship in the NICU. Infect Dis Clin North Am 2014;28:247-61.

5 Patel SJ, Oshodi A, Prasad P, et al. Antibiotic use in neonatal intensive care units and adherence with centers for disease control and prevention 12 step campaign to prevent antimicrobial resistance. Pediatr Infect Dis J 2009;28:1047-51.

6 Ting JY, Roberts A, Sherlock R, et al. Duration of initial empirical antibiotic therapy and outcomes in very low birth weight infants. Pediatrics 2019;143:e20182286.

7 Ting JY, Synnes A, Roberts A, et al. Association of antibiotic utilization and neonatal outcomes in very-low-birth-weight infants without proven sepsis. JAMA Pediatrics 2016;170:1181-7.

8 Ting JY, Synnes A, Roberts A, et al. Association of antibiotic utilization and neurodevelopmental outcomes among extremely low gestational age neonates without proven sepsis or necrotizing enterocolitis. Am J Perinatol 2018;35:972-8.

9 Cotten CM. Adverse consequences of neonatal antibiotic exposure. Curr Opin Pediatr 2016;28:141-9.

10 Cotten CM, McDonald S, Stoll B, et al. The association of thirdgeneration cephalosporin use and invasive candidiasis in extremely low birth-weight infants. Pediatrics 2006;118:717-22.

11 Neu J, Douglas-Escobar M, Lopez M. Microbes and the developing gastrointestinal tract. Nutr Clin Pract 2007;22:174-82.

12 Kuppala VS, Meinzen-Derr J, Morrow AL, et al. Prolonged initial empirical antibiotic treatment is associated with adverse outcomes in premature infants. J Pediatr 2011;159:720-5

13 Dellit TH, Owens RC, McGowan JE, et al. Infectious diseases Society of America and the Society for healthcare epidemiology of America guidelines for developing an institutional program to enhance antimicrobial stewardship. Clin Infect Dis 2007;44:159-77.

14 Patel SJ, Saiman L. Antibiotic resistance in neonatal intensive care unit pathogens: mechanisms, clinical impact, and prevention including antibiotic stewardship. Clin Perinatol 2010;37:547-63.

15 Ting J, Roberts A, Yoon E, et al. ePAS2019:2826.208 Variability and trends in antimicrobial utilization rates among infants born at $<33$ weeks' gestational age. Pediatric Academic Societies Meeting. USA: Baltimore MD, 2019.

16 Ting JY, Paquette V, Ng K, et al. Reduction of inappropriate antimicrobial prescriptions in a tertiary neonatal intensive care unit after antimicrobial stewardship care bundle implementation. Pediatr Infect Dis J 2019;38:54-9.

17 Centres for Disease Control and Prevention. Cdc 12-step program to prevent antimicrobial resistance in health care settings. Available: https://www.cdc.gov/mmwr/preview/mmwrhtml/mm5115a5.htm [Accessed 19 Apr 2002].
18 Johnson CL, Saiman L. A blueprint for targeted antimicrobial stewardship in neonatal intensive care units. Infect Control Hosp Epidemiol 2017;38:1144-6.

19 Ting JY, Shah PS. Antibiotic stewardship in neonates: challenges and opportunities. Trans/ Pediatr 2020;9:198-201.

20 Araujo da Silva AR, Marques A, Di Biase C, et al. Effectiveness of antimicrobial stewardship programmes in neonatology: a systematic review. Arch Dis Child 2020;105:563-8.

21 Douglas-Escobar M, Elliott E, Neu J. Effect of intestinal microbial ecology on the developing brain. JAMA Pediatr 2013;167:374-9.

22 Shah PS, Chan P, Yoon EY. Canadian Neonatal Network - Major anomalies list, 2013: 124-7. http://www.canadianneonatalnetwork. org/Portal/LinkClick.aspx?fileticket=|reR0871sjA\%3d\&tabid=39

23 Shah P, Yoon E, Chan P, et al. The Canadian neonatal network annual report, 2014. Available: http://www.canadianneonatalnetwork.org/ Portal/LinkClick.aspx?fileticket=eGgxmMubxjk\%3d\&tabid=39

24 Shah P, Yoon E, Chan P, et al. The Canadian neonatal network annual report, 2015. Available: http://www.canadianneonatalnetwork.org/ Portal/LinkClick.aspx?fileticket=9K3crPtfgQs\%3d\&tabid=39

25 Shah P, Yoon E, Chan P, et al. The Canadian neonatal network annual report, 2016. Available: http://www.canadianneonatalnetwork.org/ Portal/LinkClick.aspx?fileticket=PJSDwNECsMI\%3d\&tabid=39

26 Shah P, Yoon E, Chan P, et al. The Canadian neonatal network annual report, 2017. Available: http://www.canadianneonatalnetwork.org/ Portal/LinkClick.aspx?fileticket=q8BKX0wDksk\%3d\&tabid=39

27 Shirai Y, Arai H, Tamaki K, et al. Neonatal methicillin-resistant Staphylococcus aureus colonization and infection. J Neonatal Perinatal Med 2017;10:439-44.

28 Huang Y-C, Chou Y-H, Su L-H, et al. Methicillin-Resistant Staphylococcus aureus colonization and its association with infection among infants hospitalized in neonatal intensive care units. Pediatrics 2006;118:469-74.

29 Kent A, Kortsalioudaki C, Monahan IM, et al. Neonatal gramnegative infections, antibiotic susceptibility and clinical outcome: an observational study. Arch Dis Child Fetal Neonatal Ed 2016;101:F507-12

30 Tsai M-H, Chu S-M, Hsu J-F, et al. Risk factors and outcomes for multidrug-resistant gram-negative bacteremia in the NICU. Pediatrics 2014;133:e322-9.

31 Chiu C-H, Michelow IC, Cronin J, et al. Effectiveness of a guideline to reduce vancomycin use in the neonatal intensive care unit. Pediatr Infect Dis J 2011;30:273-8.

32 Calil R, Marba ST, von Nowakonski A, et al. Reduction in colonization and nosocomial infection by multiresistant bacteria in a neonatal unit after institution of educational measures and restriction in the use of cephalosporins. Am J Infect Control 2001;29:133-8.

33 Kitson AL, Rycroft-Malone J, Harvey G, et al. Evaluating the successful implementation of evidence into practice using the PARiHS framework: theoretical and practical challenges. Implement Sci 2008;3:1.

34 Kitson A, Harvey G, McCormack B. Enabling the implementation of evidence based practice: a conceptual framework. Qual Health Care 1998;7:149-58.

35 Roberts A. Using Antimicrobials Judiciously in an Age of Drug Resistance: Teaching Principles of Antimicrobial Stewardship to the next generation of Health Care Providers [program. Vancouver BC, Canada:: UBC Teaching and Learning Enhancement Fund: 2015-7.

36 Canadian neonatal network Abstracors' manual, 2015. Available: http://www.canadianneonatalnetwork.org/Portal/LinkClick.aspx? fileticket=krvGeUTtLck\%3d\&tabid=69

37 Lee SK, Shah PS, Singhal N, et al. Association of a quality improvement program with neonatal outcomes in extremely preterm infants: a prospective cohort study. CMAJ 2014;186:E485-94. 